\title{
Electron Energy-Loss Spectroscopy of Iron Nanoparticles in Lunar Soil using an Aberration-Corrected Scanning Transmission Electron Microscope.
}

\author{
M. S Thompson ${ }^{1}$ and T. J Zega ${ }^{1}$ \\ ${ }^{1}$ Lunar and Planetary Laboratory, Department of Planetary Sciences, University of Arizona, 1629 E \\ University Blvd, Tucson, AZ, 85721
}

Introduction and Motivation: Mineral grains on the surfaces of airless planetary bodies like the Moon are subjected to continuous radiation processing from solar energetic ions and also experience micrometeorite impact events. Together these processes alter the morphology, chemistry, and microstructure of surface particles and are collectively known as space weathering [1]. The optical properties of material on airless body surfaces are altered by space weathering, making it difficult to understand the chemical composition or mineralogy of that planetary body from remote sensing data [2]. Space-weathering features were first recognized in lunar soil samples returned by the Apollo missions e.g., [3]. These features primarily develop in the outer $100 \mathrm{~nm}$ of mineral grains exposed to interplanetary space, making transmission electron microscopy (TEM) uniquely suited for studying this surface process [3].

One of the primary characteristics of space weathering is the development of iron nanoparticles in the rims and interiors of mineral grains, typically $<10 \mathrm{~nm}$ in size e.g., [3]. The oxidation state of iron in these particles, termed nanophase $\mathrm{Fe}\left(\mathrm{npFe}^{0}\right)$, can provide insight into the formation conditions of these features and the overall processes governing space weathering [4]. In this study we used electron energy-loss spectroscopy (EELS) coupled to an aberration-corrected and monochromated TEM to evaluate the oxidation state of individual $\mathrm{nFFe}^{0}$ particles in lunar soils. Here we report results from six individual particles, which provide insight into the formation and processing history of grains on the surface of the Moon.

Samples and Methods: We embedded $<1 \mathrm{~mm}$ grains of Apollo lunar soil sample 79221 in a lowviscosity epoxy and prepared samples for TEM analysis through ultramicrotomy. To complete our EELS analyses we used the $100 \mathrm{keV}$ monochromated and aberration-corrected Nion UltraSTEM at Arizona State University equipped with a Gatan Enfinium spectrometer. We used a $3 \mathrm{~mm}$ spectrometer entrance aperture, a collection angle of $45 \mathrm{mrad}$ and a $0.2 \mathrm{~nm}$ probe size (with a convergence semi-angle of $30 \mathrm{mrad}$ ). Our EELS spectra for individual particles were obtained from a line profile with a 0.1 $\mathrm{eV} /$ channel dispersion and an energy resolution between 200 to $300 \mathrm{meV}$. Each measurement had a variable dwell time, ranging between $0.01 \mathrm{~s} / \mathrm{px}$ to $0.5 \mathrm{~s} / \mathrm{px}$. We collected EELS spectra for individual nanoparticles at the $\mathrm{Fe} 2,3$ core-loss edge. These spectra were compared to standards for both $\mathrm{Fe}^{0}$ and $\mathrm{Fe}^{2+}$ (expected oxidation states in these mineral samples) that had been previously acquired [4]. In order to qualitatively asses the oxidation state of $\mathrm{npFe}^{0}$, we can utilize data from previous experiments which suggest a $\sim 1.5 \mathrm{eV}$ chemical shift between $\mathrm{Fe}^{0}$ and $\mathrm{Fe}^{2+}$.

Results and Discussion: Spectra collected from the particles and reference standards are shown in Figure 1. Comparison of the particle and reference spectra shows that the L3 near-edge structure for the particles is intermediate between $\mathrm{Fe}^{0}$ and $\mathrm{Fe}^{2+}$ end members, indicating that they are composed of a mixture of oxidation states in varying proportions. Spectra a-c have primary peaks shifted to higher energies, more closely aligned with the $\mathrm{Fe}^{2+}$ standard, suggesting these particles contain more $\mathrm{Fe}^{2+}$. In 
contrast, spectra e-g have peak positions shifted to lower energies, signifying a higher proportion of $\mathrm{Fe}^{0}$ in these particles. An example of a particle from which we collected a spectrum is shown in Figure 2.

The EELS results suggest that current models for $\mathrm{npFe}^{0}$ formation may not be complete as they predict that many of these particles condense from the vapor phase in reducing conditions. Our measurements show that some particles contain oxidized Fe, which suggests that the conditions may have been more oxidizing than previously thought. However, we note that the size of the nanoparticles $(<10 \mathrm{~nm}$ on average) is smaller than the thickness of the ultramicrotomed slice $(\sim 60 \mathrm{~nm})$, and so we cannot fully rule out at this time contributions to the spectrum from surrounding matrix material in which the nanoparticles are embedded. Understanding the contribution of the matrix material to the spectra will be important for determining, conclusively, the nature of the $n \mathrm{FFe}^{0}$ oxidation states.

\section{References:}

[1] Hapke B. Journal of Geophysical Research- Planets 106 (2001), p. 10,039.

[2] Pieters C. M. et al, Meteoritics and Planetary Science 35 (2000), p. 1101.

[3] Keller L. P. and McKay D. S. Geochimica et Cosmochimica Acta 61 (1997) p. 2331.

[4] Keller L. P and Clemett S. J. Lunar and Planetary Science Conference Proceedings (2001), p. 2097.
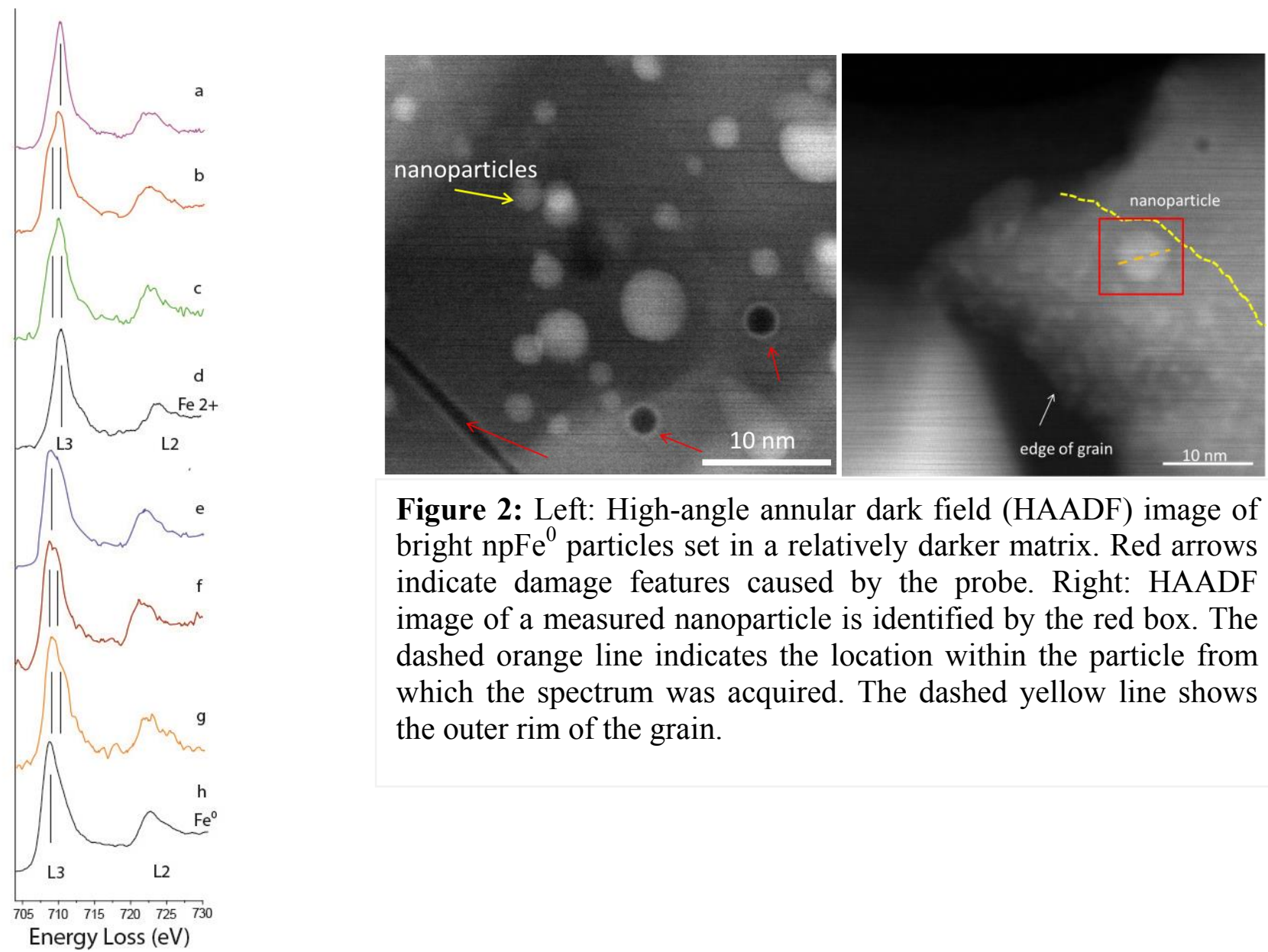

Figure 2: Left: High-angle annular dark field (HAADF) image of bright $n p \mathrm{Fe}^{0}$ particles set in a relatively darker matrix. Red arrows indicate damage features caused by the probe. Right: HAADF image of a measured nanoparticle is identified by the red box. The dashed orange line indicates the location within the particle from which the spectrum was acquired. The dashed yellow line shows the outer rim of the grain.

Figure 1: Fe L2,3 EELS spectra of six individual nanoparticles (a-c and e-g) and reference spectra for $\mathrm{Fe}^{2+}(\mathrm{d})$ and $\mathrm{Fe}^{0}(\mathrm{~h})$ from [4]. Peak positions are indicated by vertical lines. 